\title{
Molecular neuroimaging with PET/MRI
}

\author{
V. Garibotto $\cdot$ S. Förster $\cdot$ S. Haller $\cdot$ \\ M. I. Vargas $\cdot$ A. Drzezga
}

Received: 9 November 2012/ Accepted: 23 January 2013/Published online: 12 February 2013

(C) Italian Association of Nuclear Medicine and Molecular Imaging 2013

\begin{abstract}
Hybrid PET/MRI is a recently developed technique, which is attracting growing interest among the medical community owing to its potential clinical and research applications. PET/MRI is of special interest for neuroscience, given that PET and MRI are the neuroimaging methods of choice for many clinical and scientific applications. The first clinical studies conducted have tested the performance of PET/MRI in oncology indications, neurodegenerative disorders and epilepsy, using aminoacidic tracers and somatostatin-receptor imaging $\left({ }^{68} \mathrm{Ga}\right.$-DOTATOC), ${ }^{18} \mathrm{~F}$-fluorodeoxyglucose $\left({ }^{18} \mathrm{~F}-\mathrm{FDG}\right)$, and ${ }^{11} \mathrm{C}$-flumazenil, respectively, and shown that the acquisition of both brain PET and MRI can be performed in a single session on a hybrid PET/MRI system achieving quality comparable to that of PET/CT and MRI acquired separately, but with some important advantages. Combined acquisition of PET and MRI maximizes the clinical information and optimizes the
\end{abstract}

\section{Garibotto $(\square)$}

Division of Nuclear Medicine, Department of Medical Imaging and Information Sciences, Geneva University and Geneva University Hospitals, Rue Gabrielle-Perret-Gentil 4, 1211

Geneva, Switzerland

e-mail: valentina.garibotto@hcuge.ch

S. Förster

Department of Nuclear Medicine, Technische Universität München, Munich, Germany

S. Haller · M. I. Vargas

Division of Neuroradiology, Department of Medical Imaging and Information Sciences, Geneva University and Geneva University Hospitals, Rue Gabrielle-Perret-Gentil 4, 1211 Geneva, Switzerland

A. Drzezga

Department of Nuclear Medicine, University Hospital of Cologne, Cologne, Germany registration of both modalities, while minimizing patient discomfort. Sparing the patient the CT scan can reduce radiation exposure while the accurate coregistration due to the identical positioning opens new windows for better region-specific evaluation of PET data, particularly when acquired with tracers that provide little anatomical information. PET and MRI parameters can be systematically combined for diagnostic interpretation and new options concerning partial volume and motion correction can be exploited. There are still open issues, such as the selection of clinical indications, the influence of the combined design on the performance of each modality, and, in particular, the validation of quantitative PET measures with respect to attenuation correction. Overall, PET/MRI hybrid imaging is an exciting new modality and potentially the future modality of choice for neuroimaging investigations.

Keywords Hybrid imaging · PET/MRI · Neuroimaging · Brain tumors · Epilepsy $\cdot$ Neurodegenerative disorders

\section{Introduction}

Hybrid PET/MRI is a recently developed technique which is attracting growing interest among the medical community owing to its potential clinical and research applications [1-6].

Great expectations surround the use of this new modality in the field of neuroimaging [6, 7]. Indeed, PET and MRI are the methods of choice for neuroimaging, allowing combination of the molecular information (glucose metabolism for various functional investigations, aminoacid metabolism for tumor detection, receptor imaging) provided by PET imaging with the various morphological and functional parameters measured by MR. 
In this respect, MRI, due to its superior soft-tissue contrast, has clear advantages over CT. In particular, MRI clearly outperforms non-contrast-enhanced CT (which is usually coupled to PET in routine brain PET/CT investigations), without causing radiation exposure and even when no contrast media are employed. Furthermore, more than with CT, various functional and molecular insights can be derived from MRI. Thus, it appears plausible that the systematic combination of PET with MRI may offer completely new opportunities for research applications and for molecular imaging of the brain in general.

The results of coregistration of PET and MRI data, routinely done for brain imaging, prove the added value of multimodality imaging, but this value is affected by the need to position the patient in different scanners, often in different physiological conditions and at different times. These problems are potentially solved by hybrid PET/MRI systems that combine the excellent soft-tissue contrast at high resolution of MRI, together with its additional imaging options such as spectroscopy, functional MRI, diffusion- and perfusion-weighted imaging, with the molecular, biochemical, and functional information provided by PET. For this reason, as well as for technical reasons (size of the PET insert), the first prototype tested in humans was a brain-dedicated system [3].

This review will describe the results of the brain PET/ MRI studies performed so far using integrated systems and will discuss the potential role played by this technique in the field of molecular neuroimaging, together with challenges and future perspectives.

\section{Methods}

A literature search was conducted using the Medline database. General inclusion criteria included research paper and review articles published in English in peer-reviewed journals. The keywords, used in PubMed, were the following: hybrid PET/MRI, neuroimaging, molecular imaging. For completeness, additional specific papers known to the authors that did not meet the initial search criteria were also included.

\section{PET/MRI technology and neuroimaging}

Considerable effort has been put into the development of combined PET and MRI, and the design of currently available systems reflects different solutions proposed to overcome the major challenges arising on bringing these two technologies together [8]. First, for PET detectors to be compatible with the MRI magnetic field, photomultiplier technology must be replaced with magnetic field- insensitive avalanche photodiodes or silicon photomultipliers [9, 10]. Alternatively, sequential imaging and proper shielding can be used to minimize magnetic effects on the PET electronics [4, 5].

The design of the hybrid PET/MRI tomographs is described in detail elsewhere in this issue.

For hybrid neuroimaging, both brain-dedicated systems and whole-body systems have been tested as described in detail in the following sections.

\section{Brain-dedicated PET/MRI tomographs}

A prototype for simultaneous PET/MRI of the brain featuring a PET insert for a standard $3 \mathrm{~T}$ MRI scanner (BrainPET; Siemens) was the first successful approach showing the potential of truly simultaneous PET/MRI performed in humans [3].

Two studies have also shown the feasibility of a research prototype consisting of a shuttle bed-based combination of ultra-high-field MRI with high-resolution research tomograph (HRRT) PET for brain applications [11, 12]. The system components represent the high end of both technologies, namely MRI at $7 \mathrm{~T}$, allowing enhanced sensitivity and resolution, and HRRT PET, providing high sensitivity and spatial resolution thanks to the physical and geometrical characteristics of the scanner detectors. These two brain-dedicated systems are connected via a rotating shuttle bed with precision calibration [11, 12].

Whole-body PET/MRI tomographs

A decade after the commercial introduction of PET/CT, whole-body PET/MRI scanners have now been introduced on the market for clinical use. Whole-body PET/MRI scanners, able to perform both brain and whole-body imaging, can be expected to become more widely available over the next few years, whereas dedicated brain systems will presumably have limited availability, mainly confined to research centers.

Commercial solutions for combined PET and MRI are currently available from three vendors. GE Healthcare proposes a system with PET/CT and MRI scanners placed in neighboring rooms, with the ability to shuttle a patient bed between the rooms; each scanner can be used separately as a stand-alone system as well, or they can be used consecutively on the same patient, resulting in a trimodality approach [13].

Philips Healthcare offers a sequential PET/MRI scanner, called Ingenuity TF PET/MR, which combines a 3 T MRI scanner and a PET scanner with time-of-flight (TF) technology. The PET and MRI scanners are placed beside each other in a single room, and a patient bed capable of rotating 180 degrees is used to transfer the patient from one scanner 
into the other [14, 15]. PET and MRI images are acquired sequentially, in analogy to what is currently done in PET/ CT systems. Some recent publications have already reported on the use of the Philips Ingenuity TF PET/MRI tomograph with regard to clinical applications in neurology [16-19].

Siemens Healthcare offers Biograph mMR, the first commercially available integrated PET/MRI whole-body scanner, in which, thanks to the use of MRI-compatible PET photodetectors, the PET detector ring is placed within the MRI main magnet, allowing simultaneous acquisition of the two modalities [20].

The first results of brain PET/MRI studies obtained on the integrated Biograph mMR whole-body PET/MRI system are already discussed in a report on workflow considerations for whole-body staging of oncology patients [21].

The design of a hybrid tomograph also has an impact on cost-efficacy considerations, as recently discussed [22]. The cost-efficacy of a hybrid modality vs separate systems depends on the costs of each component in separate and integrated systems, on exam duration, and on patient changeover times, but cost-efficacy analyses should also take into account the diagnostic gain that can be expected from the combination of different modalities. This complex and important topic needs to be addressed in detail in dedicated studies, with specific reference to brain as well as whole-body imaging applications which is outside the scope of the current article.

\section{Clinical applications of PET/MRI molecular neuroimaging}

Recent published studies on clinical applications of PET/ MRI neuroimaging include the following topics: tumors, vascular disorders, neurodegenerative disorders, and epilepsy. Table 1 summarizes the studies published so far on each of these topics. The results are described in more detail in the following paragraphs.

\section{Tumors}

Because of its high soft-tissue contrast, MRI has become the first-line method of choice for the assessment of brain tumors. Although conventional sequences allow excellent visualization of these tumors, they still have limitations as regards precise definition of lesion boundaries and the monitoring of treatment-related changes and tumor recurrence. Advanced MRI techniques such as diffusionweighted imaging, perfusion-weighted imaging, and MR spectroscopy provide a better estimation of tumor extension and potentially higher accuracy in tumor grading $[23,24]$.
Table 1 Summary of the integrated PET/MRI studies currently available, grouped according to clinical indication

\begin{tabular}{llll}
\hline Clinical indication & PET tracer & $\begin{array}{l}\text { No. of } \\
\text { patients } \\
\text { investigated }\end{array}$ & References \\
\hline $\begin{array}{l}\text { Functional assessment } \\
\text { for vascular and/or } \\
\text { neurodegenerative }\end{array}$ & ${ }^{18}$ F-FDG & 4 & {$[16]$} \\
cognitive disturbances & & 1 & {$[17]$} \\
Epileptic focus & ${ }^{18}$ F-FDG & 6 & {$[30]$} \\
localization & ${ }^{11}$ C- & 1 & {$[16]$} \\
& flumazenil & & {$[27]$} \\
Brain tumors & & & \\
Gliomas & ${ }^{18}$ F-FET & 5 & {$[16]$} \\
Gliomas & & 4 & {$[28]$} \\
& ${ }^{11}$ C- & 7 & {$[29]$} \\
Meningiomas & methionine & 14 & {$[30]$} \\
& ${ }^{68}$ Ga- & 3 & {$[29]$} \\
Metastatic lesions & DOTATOC & 8 & {$[30]$} \\
\hline
\end{tabular}

PET can provide important biochemical and metabolic information in addition to the morphological, functional, and metabolic information obtainable by MRI. In particular, PET with specific amino acid tracers, such as ${ }^{11} \mathrm{C}$-methionine and ${ }^{18} \mathrm{~F}$-fluoroethyl-L-thyrosine $\left({ }^{18} \mathrm{~F}\right.$-FET), is now an established diagnostic tool in the clinical management and treatment planning of cerebral gliomas. In diagnostic assessment and staging, this modality can be used to guide biopsy in heterogeneous tumors; furthermore, it may help to determine tumor extent and to differentiate it from the edema typically surrounding a mass lesion, and it may also be useful in guiding the choice of treatment. Furthermore, PET imaging with amino acid tracers can help to distinguish post-therapeutic alterations from tumor recurrence $[25,26]$.

Thus, combined amino acid PET and MRI is likely to lead to enhanced diagnostic sensitivity for gliomas and may allow a closer correlation between tracer uptake and perfusion and metabolic changes shown by MRI in neoplastic tissue. In general, the combination of PET and MRI may lead to better characterization of tumor tissue and better differentiation between tumor tissue and therapyinduced changes in the brain. With respect to therapyinduced changes, multimodal PET and MR imaging might play an important role, offering better characterization of changes after anti-angiogenic therapies and radiation necrosis. The development of new specific tracers will presumably have a strong impact on this field and the hybrid tomographs will be the ideal instruments to test the combined performance of PET and MRI modalities. 
As summarized below, various specific tracers have been tested in PET/MRI hybrid scanners to evaluate their potential to help characterize intracranial masses.

\section{${ }^{18}$ F-FET}

A small series of patients, including adult and pediatric patients with high-grade brain tumors, underwent ${ }^{18} \mathrm{~F}$-FET PET/MRI. In this study, good image quality of both modalities was obtained for all subjects [16]. PET/MRI images obtained with this tracer were also acquired simultaneously in a small population of glioma patients in a comprehensive one-stop examination lasting a total of 50 min $[27,28]$. Two examples of hybrid PET/MRI images are shown in Fig. 1.

\section{${ }^{11}$ C-methionine}

Two papers described the performance, in glioma patients (including both low- and high-grade lesions), of
Fig. 1 Tumor imaging with hybrid PET/MRI. ${ }^{18}$ F-FET PET/ MRI of a recurrent grade III oligodendroglioma (a study acquired on a Philips Ingenuity TF PET/MRI, Hôpitaux Universitaires de Genève, Geneva, Switzerland) and in a multifocal glioblastoma (b study acquired on the Biograph mMR whole-body, Technische Universitat Munchen, Munich, Germany). The added value of hybrid PET/ MRI is the distinction between recurrence and scar in $\mathbf{a}$, and the observation of significant PET uptake in both lesions in $\mathbf{b}$, despite different Gd uptake patterns

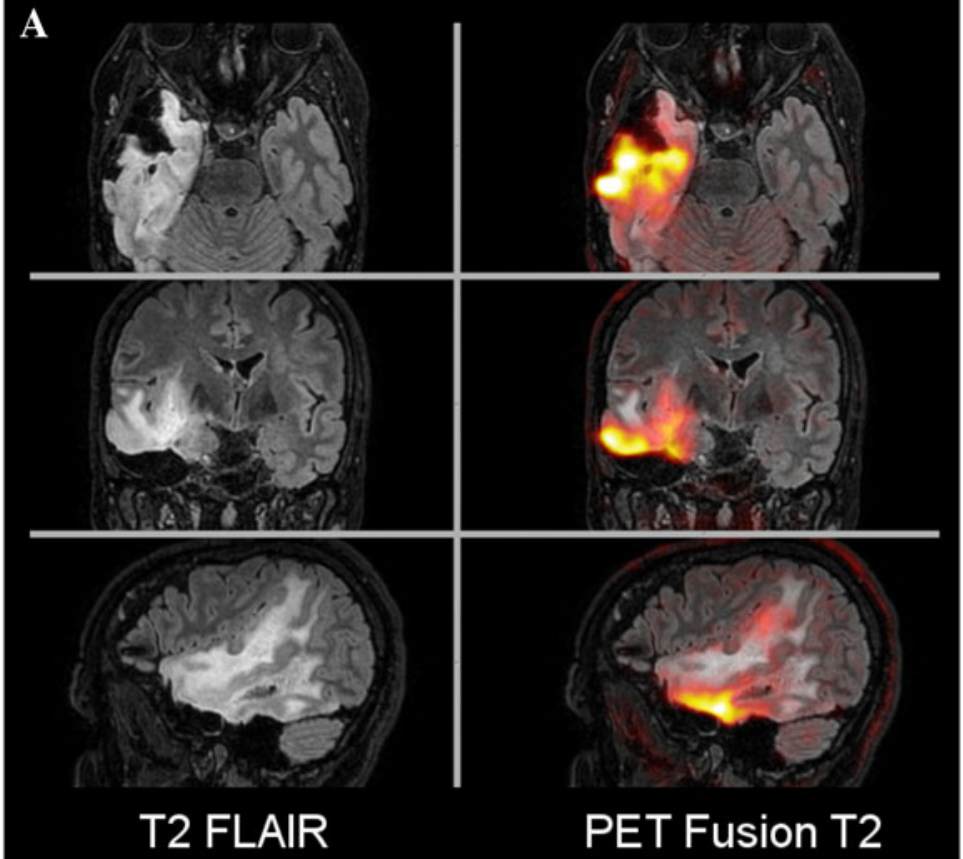

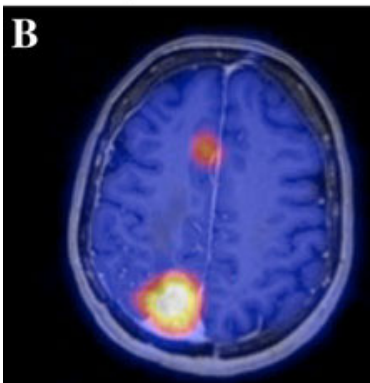
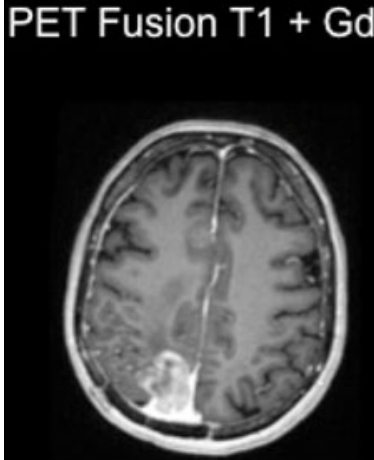

$\mathrm{T} 1+\mathrm{Gd}$

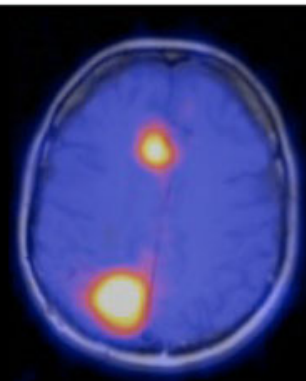

PET Fusion T1

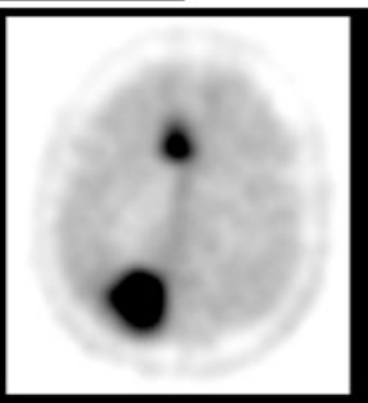

PET 
simultaneous PET/MRI, as compared with PET/CT, performed using the tracer ${ }^{11} \mathrm{C}$-methionine $[29,30]$. The two studies, by the same group, investigated subjects with brain tumor, mainly astrocytomas, by PET/CT and subsequently simultaneous PET/MRI (dedicated brain system); no significant difference was found in the visual classification of positive/negative studies and quantitative ratios to reference tissues were comparable [29, 30]. In addition, overall the quality of MRI was good: only a small percentage (about $10 \%$ ) of subjects showed major artifacts on some MR sequences and there was only one non-diagnostic echo planar sequence [30].

\section{${ }^{68} \mathrm{Ga}-\mathrm{DOTATOC}$}

The two abovementioned papers also compared the results obtained with PET/CT and PET/MRI, using the tracer ${ }^{68} \mathrm{Ga}$-DOTATOC, in meningioma patients: they demonstrated that image quality and quantitative data obtained with the two modalities have good agreement [29, 30]. Importantly, in two patients the authors identified an additional lesion in the PET/MRI dataset, which was not identified on PET/CT images [30]. The use of this new modality for intensity-modulated radiation therapy has also been tested, in one patient, by Thorwarth et al. [31]. The patient, presenting a recurrence of an atypical meningioma after surgical resection, was studied by PET/CT and subsequently by simultaneous PET/MRI (dedicated brain system). The PET image obtained using the hybrid system identified an additional small tumor region, as compared with the PET/CT dataset: this difference is presumably due to the improved spatial resolution of this brain-dedicated system, even though other differences between the two datasets, such as uptake time, voxel size, and attenuation correction procedure, may confound the result [31].

\section{${ }^{18}$ F-FDG for metastatic brain lesions}

Given the high physiological uptake of ${ }^{18}$ F-FDG in the cortex, small brain metastases cannot be reliably ruled out in a whole-body PET/CT evaluation. For this reason, an additional brain MRI is required for staging if the risk of brain metastases is high. Hybrid PET/MRI, when performed for whole-body staging, has a potential advantage over PET/CT in this domain. Recent data show that whole-body MRI protocols have a sensitivity that is lower than that of dedicated MRI brain scans, but still superior to that of ${ }^{18} \mathrm{~F}-\mathrm{FDG}$ PET/CT studies $[32,33]$. To the best of our knowledge, data on the diagnostic performance of integrated PET/MRI for the detection of cerebral metastases are not available at present. The patient sample studied by Schwenzer et al. [30] includes six brain metastases studied using ${ }^{18}$ F-FDG (reported in Table 1), but no detailed information concerning the diagnostic performance of PET/MRI vs PET/CT for staging of secondary brain lesions is provided.

Neurodegenerative and cerebrovascular disorders

Brain imaging tools are among the most promising candidate instruments in the search for sensitive biomarkers of dementia and other neurodegenerative disorders. In particular, ${ }^{18}$ F-FDG PET as a marker of regional neuronal function is a well-established sensitive and specific method for early identification of Alzheimer's disease (AD) pathology, for differential diagnosis, and for prediction of $\mathrm{AD}$, even in the mild and asymptomatic stages [34].

A recent study, in a small group of patients with neurodegenerative and vascular disorders, investigated the performance of hybrid PET/MRI, using ${ }^{18}$ F-FDG as the PET tracer. A superior specificity of MRI for vascular lesions was found. Conversely, a superior specificity of PET metabolic patterns was observed with regard to accurate diagnosis of Lewy body dementia and frontotemporal dementia (validated by clinical diagnosis at follow-up) [16]. Figure 2 shows a representative case of frontotemporal dementia with concordant PET and MRI data. This finding is consistent with previous literature data [35, 36].

Schwenzer et al. [30], also using ${ }^{18}$ F-FDG as the PET tracer, performed simultaneous PET/MRI following PET/ CT in 11 patients with neurodegenerative disorders (7 patients with Parkinson's disease, 2 with $\mathrm{AD}$ and 2 with progressive supranuclear palsy). They confirmed a high agreement of PET results between PET/MRI and PET/CT and demonstrated that simultaneous PET/MRI acquisition resulted in adequate MRI quality.

\section{Epilepsy}

Interictal ${ }^{18} \mathrm{~F}$-FDG PET studies are usually performed to identify hypometabolic foci, which might represent dysfunctional epileptogenic cortex, even in MRI-negative cases, or in cases with multiple MRI lesions [37]. ${ }^{11} \mathrm{C}$-flumazenil PET, a ligand for the inhibitory gamma-aminobutyric acid receptors, is also able to show focal reductions in epileptogenic cortex [37]. There is strong evidence that combined PET and MRI imaging can be recommended for a complete evaluation of epilepsy: previous reports consistently demonstrated the added value of software fusion of PET and MRI data for the pre-surgical evaluation of cortical dysplasia $[38,39]$.

To date, only a few PET/MRI studies on epilepsy have been reported $[16,27]$. One report on six cases studied by sequential PET/MRI hybrid imaging showed that fusion of morphological and functional information allowed the identification of subtle metabolic alterations which could easily have been missed when interpreting the ${ }^{18} \mathrm{~F}$-FDG images alone [16]. Figure 3 shows images of a PET/MRI 

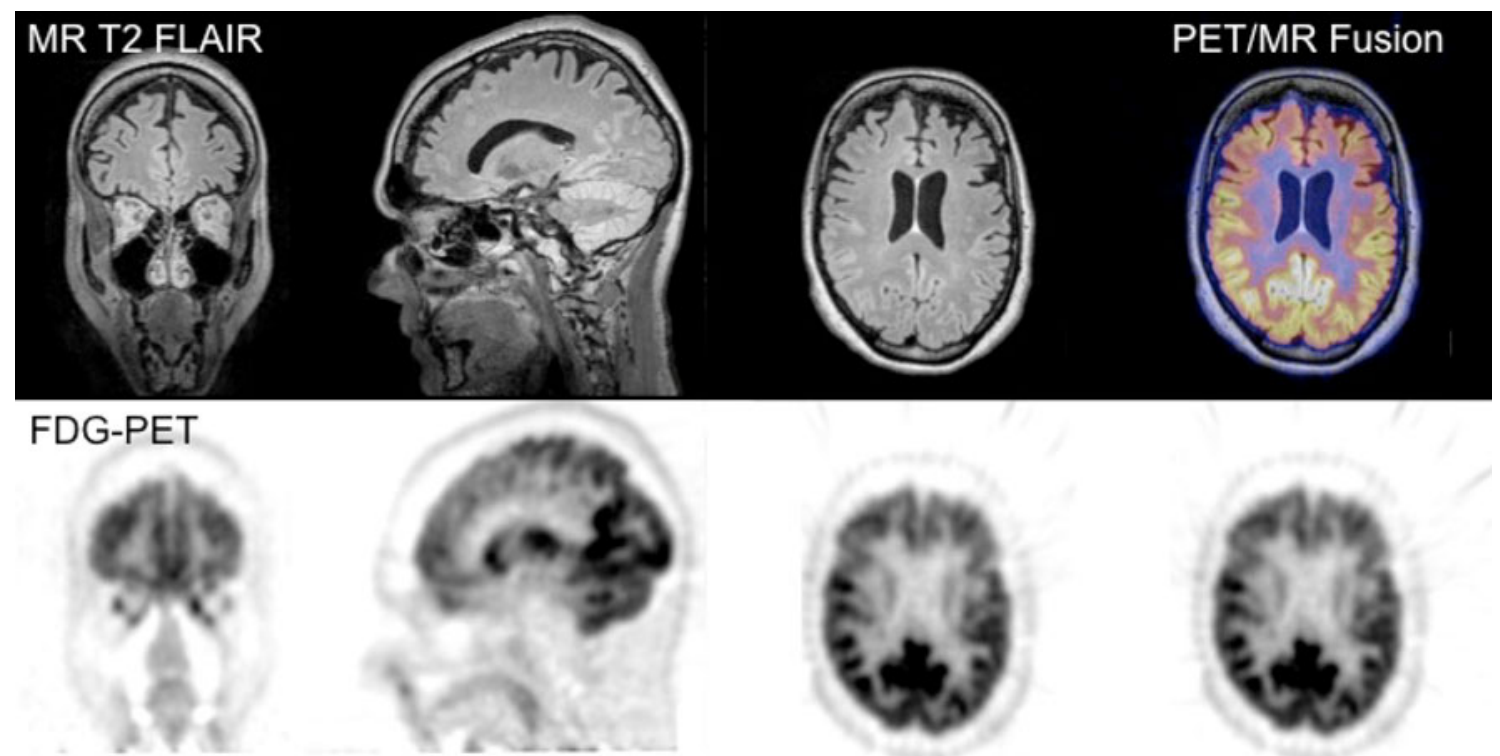

Fig. 2 Imaging of neurodegeneration by PET/MRI. ${ }^{18}$ F-FDG PET/ MRI study in a patient with clinical suspicion of degenerative dementia: the images show concordant information on both modalities, i.e. diffuse atrophy on MRI and significant hypometabolism in

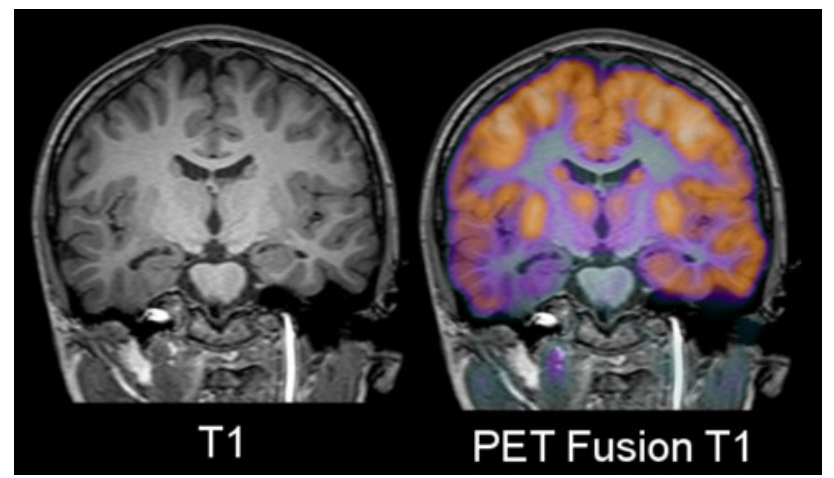

Fig. 3 Imaging epilepsy by PET/MRI. ${ }^{18}$ F-FDG PET/MRI study showing concordant information on the two modalities, i.e. right temporal hypometabolism associated with hippocampal sclerosis and amygdalo-hippocampal dysplasia in a pediatric patient (study acquired on a Philips Ingenuity TF PET/MRI, Geneva University Hospitals, Geneva, Switzerland)

study in a pediatric case. Another study demonstrated the feasibility of simultaneous ${ }^{11} \mathrm{C}$-flumazenil PET and MPRAGE MRI acquisition in one patient with right temporal lobe epilepsy [27].

\section{PET/MRI molecular neuroimaging in neuroscience research}

Integrated PET/MRI might have an important role to play in neuroscience research, particularly for multi-parametric analysis of complex functions in neural networks.
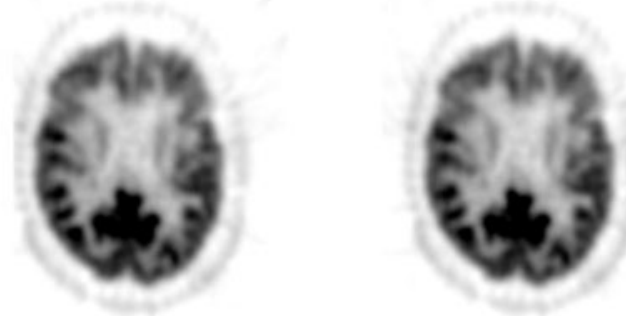

frontal regions, typical of frontotemporal dementia (study acquired on the Biograph mMR whole-body, Technische Universitat Munchen, Munich, Germany)

Molecular neuroimaging research is currently based on systematic association of PET and MRI findings by software coregistration, combining the multiple parameters measured by both modalities.

One problem of separate acquisitions, which is overcome by integrated systems, is the fact that the PET and MRI acquisitions are often performed on separate days, leading to possible differences in the physiopathological conditions, depending on the population and on the process being evaluated.

A specific advantage of simultaneous systems is that fastchanging processes in the brain can be evaluated by both modalities at the same time. This directly concerns only a small proportion of neuroscience research performed using PET: indeed, most PET studies evaluate steady-state conditions for which simultaneity of the processes being evaluated is not an issue. However, for those studies evaluating neurotransmitter release, the simultaneous design makes it possible to combine this measure with changes in bloodoxygen-level-dependent (BOLD) signal measured by fMRI. This perspective, interesting and exciting, has been evoked in many previous review papers $[6,7]$ but, to the best of our knowledge, has not yet been tested in human studies. There may be various reasons for this: hybrid PET/MRI technology is quite recent and most of the studies performed so far have focused mainly on validating and optimizing its clinical use. In addition, the quantitative validation of MRI-based attenuation correction (see below) is still ongoing and is a prerequisite for quantitative imaging, such as receptor imaging, with this new modality. 
Advantages of the hybrid PET/MRI modalities in brain imaging

Single imaging session

A main advantage of hybrid imaging technology is the fact that all relevant imaging information can be collected in a single session, simultaneously or sequentially, depending on the tomograph design (as described in Sect. 3).

In addition, while a PET/MRI study is longer than a standard diagnostic PET/CT study, it is still shorter than the total time that a PET/CT and an MRI study would take when performed on two separate scanners at different times, since the patient only has to be positioned once or, when available, synchronous acquisition can be performed. This issue is particularly important for patients with limited compliance, having some degree of executive and motor impairment, as is often the case with patients investigated for neurodegenerative disorders or brain lesions.

In addition, the single imaging session, as compared with two separate sessions, is more comfortable and timeefficient for patients and caregivers, and this is especially important when additional procedures, such as anesthesia or sedation, are required.

Multimodal image acquisition at one time point also guarantees that all conditions, in particular disease stage and effects of treatment (e.g. radiation therapy, medication, etc.), are the same for the two studies. This is not necessarily the case for separately acquired PET and MRI scans, which are often performed some days if not weeks apart. Given the usually busy clinical schedules at PET/CT and MRI units, it can in general be very difficult, outside a dedicated research environment, to obtain both a PET and an MRI scan in rapid succession.

\section{Lower radiation exposure}

A factor favoring the use of MRI over CT in hybrid imaging devices is the fact that avoiding CT means avoiding submitting the patient to an additional radiation dose, from the use of ionizing radiation. The contribution of CT imaging in PET/CT examinations has been estimated to range from 20 to $450 \mu \mathrm{Sv}$, depending on whether a lowdose or a high-quality diagnostic brain scan is used [40].

The reduced radiation exposure from PET/MRI compared with PET/CT is of special interest in the pediatric population $[41,42]$ and in cases requiring repeated investigations, obviously.

\section{Integration of PET and MRI parameters}

PET and MRI information can be used in synergy for image interpretation; furthermore, they can also be combined quantitatively: e.g. MRI parameters, such as regional perfusion or regional gray matter volume, might be used to obtain more precise quantification of PET parameters, such as standard uptake values and distribution volumes, as summarized below.

\section{Partial volume correction of PET data}

The correction for partial volume effects (PVE) is a wellknown technique for PET quantification which tries to overcome the relatively limited spatial resolution of PET by taking into account the size of the structure emitting the PET signal. MRI can be used to estimate the structural framework underlying the PET signal emitted from the brain [43]. Correction for partial volume effects has a special relevance for conditions such as neurodegeneration, which are associated with a considerable degree of atrophy. PET/MRI tomographs have the possibility to provide both "standard" and "PVE-corrected" images. The respective diagnostic value has still to be tested, but a gain in diagnostic performance is expected. Combined PET and MR information will help to assess the occurrence of atrophy, of metabolic disturbances, or both. Recent work has proven that these processes may be present in different regions at different disease stages but not necessarily to the same extent, e.g. in AD [44]. The accuracy of PVE correction methods is affected by many factors and none is widely adopted in clinical practice, as recently summarized [6].

\section{Correction for perfusion or blood-brain barrier disruption for PET quantification}

Different MRI techniques allow estimation of various parameters associated with regional cerebral blood supply/ distribution, such as contrast-enhanced MRI, arterial spin labeling, and diffusion imaging [45].

Regional perfusion is a parameter of the utmost importance for PET quantification because it reflects the delivery of tracer to binding sites (defined as the "input function"). The combination of PET and MRI in a single session could therefore allow the acquired PET data to be complemented with an input function measured non-invasively by MRI. Obviously, this will only be possible if this approach can be accurately validated against a "gold standard" measure of perfusion, e.g. using PET imaging with ${ }^{15} \mathrm{O}$-water. Integrated PET/MRI in a hybrid tomograph allowing simultaneous acquisitions may have a specific added value in this respect, allowing the comparison of two different measures of the same parameter obtained by MRI and PET in precisely the same experiment.

The distribution of many PET tracers in the brain is influenced not only by perfusion-dependent regional tracer 
supply but also by the integrity of the blood-brain barrier. Contrast-enhanced MRI allows the assessment of bloodbrain disruption. Thus, combining measurement of PET binding with regional perfusion/contrast enhancement might allow correction of the PET data for effects of blood-brain barrier disruption, which can otherwise lead to false-positive judgment of tracer uptake in tumors or inflammatory brain lesions [46].

\section{Motion correction}

This is a specific opportunity offered by systems acquiring PET and MRI images simultaneously: given the temporal correlation of the signals from the two modalities, the MRI information can be used for PET data motion correction (MC). Subject motion might be difficult to avoid and can lead to degradation of PET images and severe artifacts when motion has large amplitude. This is of particular relevance for patients who suffer from movement disorders or whose collaboration is limited (pediatric cases, patients with cognitive impairment). An MC algorithm based on high-temporal-resolution MRI-derived motion estimates (obtained while simultaneously acquiring anatomical or functional MRI data) was recently validated in phantom studies, studies in healthy volunteers, and preliminary studies in patients with $\mathrm{AD}[47,48]$.

\section{Challenges and open questions}

Clinical indications: when is PET/MRI the brain examination of choice?

PET/MRI will potentially become the examination of choice for most clinical applications of brain molecular imaging, once it is available. As mentioned above, this includes oncology imaging, and imaging of neurodegenerative conditions and epilepsy. This assumption is based on the fact that both modalities provide independent and complementary information with high sensitivity and specificity in these pathological brain conditions. In addition, the availability of both modalities in one examination will encourage the co-interpretation of the two signals: the need for multidisciplinary and consensual reading may in itself represent an added value.

When hybrid tomography is not available, it will always be possible to perform a post hoc fusion of brain images acquired on separate scanners, as is currently done in most centers, thanks to the large availability of software solutions for post hoc fusion of brain images. This works well in principle, because the brain as a non-moving organ represents a rigid body; thus, coregistration of images derived from different modalities can be performed reliably. However, some PET tracers provide only very limited anatomical information. This includes tracers showing binding only to specific brain structures, e.g. imaging of the basal ganglia with ${ }^{18} \mathrm{~F}$-fluorodopa. Other tracers, e.g. for amyloid plaque imaging, show binding only to specific lesions and no binding in subjects who do not express the corresponding pathology. Combined PET/ MRI acquisition in a hybrid scanner may considerably improve the precision of anatomical evaluation of PET data acquired with these tracers. Furthermore, as mentioned above, exact temporal and regional correlation of PET and MR data acquired at exactly the same time in exactly the same position carries a number of advantages.

\section{Exam duration}

Depending on the design of the system and on the protocol adopted, mainly for the realization of MRI images, the PET/MRI examination may still be relatively long, even on a hybrid system [18]. In this respect, tomographs performing simultaneous acquisition might be advantageous. A long exam duration might result in patient movement and motion artifacts, especially for patients only partially able to collaborate or tolerate immobilization. In the future, systematic combined acquisition of PET and MR data may make it possible to establish whether some of the information obtained by either of the methods is redundant and can be done without to reduce examination time.

\section{PET and MRI performance}

Validation studies have been performed to quantify the relative impact of the integrated design on each system, as compared with their functioning as independent systems. Preliminary results show that the quality of PET and MRI is adequate [14, 15, 49-51]. A detailed description of these methodological issues is beyond the scope of this review. One aspect, however, the quantification of the PET data using MRI-based attenuation correction, has major relevance for brain studies. Several solutions for attenuation correction of the PET/MRI data have been proposed, e.g. atlas-based methods or MR-based segmentation of the scanned volume into specific tissue types with known attenuation factors [52]. The solution adopted by the current PET/MRI integrated systems is based on the acquisition of a Dixon MRI sequence which allows segmentation of the scanned volume into four tissue types (water, lungs, fat, background/air). On the basis of this segmented MR data an estimated attenuation correction map is calculated. This map necessarily represents an approximation as compared with attenuation maps based on germanium sources or computed tomography imaging, as used for correction of conventional PET and PET/CT scans. 
Furthermore, this solution does not account for attenuation induced by bone. This may represent a specific problem for the quantification of emission from the brain, given that it is surrounded by bone. Thus, other solutions, such as dualecho MR sequences which allow an approximation of bone distribution, are currently under evaluation, with ongoing studies focusing on optimization of MR-based attenuation correction and a precise understanding of the impact of this issue on quantitative PET measurement $[53,54]$.

\section{Conclusions}

The possibility of combining PET with a large variety of MRI sequences during a single examination opens new perspectives for bimodal multi-parametric imaging of the human brain. Acquiring both PET and MRI in a single session on a hybrid tomograph is expected to improve both clinical diagnosis and patient comfort. Integrated PET/MRI is likely to become more broadly available in the future, and it can be expected that the results obtained by the groups currently working with this method in clinical routine will help in the identification of the best suited clinical and research applications and in the adoption of simplified PET and MRI protocols for shorter and more informative clinical examinations.

Finally, PET/MRI may represent an ideal tool for in vivo brain research and the combined use of the two techniques may improve understanding of the physiological and pathophysiological phenomena underlying brain functioning and brain disorders.

Conflict of interest This work was supported by the Aetas Swiss Foundation for Ageing Research and by the German Research Foundation (DFG). Prof. Drzezga has received honoraria from Siemens Healthcare and GE Healthcare for lectures. The other authors of this article have no relevant relationships that could be perceived as real or apparent conflicts of interest.

\section{References}

1. Ratib O, Beyer T (2011) Whole-body hybrid PET/MRI: ready for clinical use? Eur J Nucl Med Mol Imaging 38:992-995. doi: 10.1007/s00259-011-1790-4

2. Boss A, Stegger L, Bisdas S, Kolb A, Schwenzer N, Pfister M, Claussen CD, Pichler BJ, Pfannenberg C (2011) Feasibility of simultaneous PET/MR imaging in the head and upper neck area. Eur Radiol 21:1439-1446. doi:10.1007/s00330-011-2072-z

3. Schlemmer HP, Pichler BJ, Schmand M, Burbar Z, Michel C, Ladebeck R, Jattke K, Townsend D, Nahmias C, Jacob PK, Heiss WD, Claussen CD (2008) Simultaneous MR/PET imaging of the human brain: feasibility study. Radiology 248:1028-1035. doi: 10.1148/radiol.2483071927

4. Beyer T, Pichler B (2009) A decade of combined imaging: from a PET attached to a CT to a PET inside an MR. Eur J Nucl Med Mol Imaging 36(Suppl 1):S1-S2. doi:10.1007/s00259-0081041-5
5. Zaidi H, Del Guerra A (2011) An outlook on future design of hybrid PET/MRI systems. Med Phys 38:5667-5689. doi:10.1118/ 1.3633909

6. Catana C, Drzezga A, Heiss WD, Rosen BR (2012) PET/MRI for neurologic applications. J Nucl Med 53:1916-1925. doi:10.2967/ jnumed.112.105346

7. Heiss WD (2009) The potential of PET/MR for brain imaging. Eur J Nucl Med Mol Imaging 36(Suppl 1):S105-S112. doi: 10.1007/s00259-008-0962-3

8. Slates RB, Farahani K, Shao Y, Marsden PK, Taylor J, Summers PE, Williams S, Beech J, Cherry SR (1999) A study of artefacts in simultaneous PET and MR imaging using a prototype MR compatible PET scanner. Phys Med Biol 44:2015-2027

9. Judenhofer MS, Wehrl HF, Newport DF, Catana C, Siegel SB, Becker M, Thielscher A, Kneilling M, Lichy MP, Eichner M, Klingel K, Reischl G, Widmaier S, Rocken M, Nutt RE, Machulla HJ, Uludag K, Cherry SR, Claussen CD, Pichler BJ (2008) Simultaneous PET-MRI: a new approach for functional and morphological imaging. Nat Med 14:459-465. doi:10.1038/nm1700

10. Catana C, Wu Y, Judenhofer MS, Qi J, Pichler BJ, Cherry SR (2006) Simultaneous acquisition of multislice PET and MR images: initial results with a MR-compatible PET scanner. J Nucl Med 47:1968-1976. doi:10.1073/pnas.0711622105

11. Cho ZH, Son YD, Choi EJ, Kim HK, Kim JH, Lee SY, Ogawa S, Kim YB (2012) In vivo human brain molecular imaging with a brain-dedicated PET/MRI system. MAGMA. doi:10.1007/ s10334-012-0329-4

12. Cho ZH, Son YD, Kim HK, Kim KN, Oh SH, Han JY, Hong IK, Kim YB (2008) A fusion PET-MRI system with a high-resolution research tomograph-PET and ultra-high field 7.0 T-MRI for the molecular-genetic imaging of the brain. Proteomics 8:1302-1323. doi:10.1002/pmic.200700744

13. Veit-Haibach P, Kuhn FP, Wiesinger F, Delso G, von Schulthess G (2012) PET-MR imaging using a tri-modality PET/CT-MR system with a dedicated shuttle in clinical routine. MAGMA. doi: 10.1007/s10334-012-0344-5

14. Zaidi H, Ojha N, Morich M, Griesmer J, Hu Z, Maniawski P, Ratib O, Izquierdo-Garcia D, Fayad ZA, Shao L (2011) Design and performance evaluation of a whole-body Ingenuity TF PETMRI system. Phys Med Biol 56:3091-3106. doi:10.1088/00319155/56/10/013

15. Kalemis A, Delattre BM, Heinzer S (2012) Sequential wholebody PET/MR scanner: concept, clinical use, and optimisation after two years in the clinic. The manufacturer's perspective. MAGMA. doi:10.1007/s10334-012-0330-y

16. Garibotto V, Heinzer S, Vulliemoz S, Guignard R, Wissmeyer M, Seeck M, Lovblad KO, Zaidi H, Ratib O, Vargas MI (2013) Clinical applications of hybrid PET/MRI in neuroimaging. Clin Nucl Med 38:e13-e18. doi:10.1097/RLU.0b013e3182638ea6

17. Garibotto V, Vargas MI, Lovblad KO, Ratib O (2011) A PETMRI case of corticocerebellar diaschisis after stroke. Clin Nucl Med 36:821-825. doi:10.1097/RLU.0b013e31821a27f8

18. Vargas MI, Becker M, Garibotto V, Heinzer S, Loubeyre P, Gariani J, Lovblad KO, Vallee JP, Ratib O (2012) Approaches for the optimization of MR protocols in clinical hybrid PET/MRI studies. MAGMA

19. Vargas MI, Garibotto V, Viallon M, Guignard R, Cuvinciuc V, Lovblad KO, Ratib O (2013) Peripheral nerves, tumors and hybrid PET-MRI. Clin Nucl Med 38:e40-e42. doi:10.1097/RLU. 0b013e31824c6013

20. Drzezga A, Souvatzoglou M, Eiber M, Beer AJ, Furst S, MartinezMoller A, Nekolla SG, Ziegler S, Ganter C, Rummeny EJ, Schwaiger M (2012) First clinical experience with integrated wholebody PET/MR: comparison to PET/CT in patients with oncologic diagnoses. J Nucl Med 53:845-855. doi:10.2967/jnumed.111. 098608 
21. Martinez-Moller A, Eiber M, Nekolla SG, Souvatzoglou M, Drzezga A, Ziegler S, Rummeny EJ, Schwaiger M, Beer AJ (2012) Workflow and scan protocol considerations for integrated whole-body PET/MRI in oncology. J Nucl Med doi:10.2967/ jnumed.112.109348

22. von Schulthess GK, Burger C (2010) Integrating imaging modalities: what makes sense from a workflow perspective? Eur J Nucl Med Mol Imaging 37:980-990. doi:10.1007/s00259-0091378-4

23. Khayal IS, McKnight TR, McGue C, Vandenberg S, Lamborn KR, Chang SM, Cha S, Nelson SJ (2009) Apparent diffusion coefficient and fractional anisotropy of newly diagnosed grade II gliomas. NMR Biomed 22:449-455. doi:10.1002/nbm.1357

24. Noguchi T, Yoshiura T, Hiwatashi A, Togao O, Yamashita K, Nagao E, Shono T, Mizoguchi M, Nagata S, Sasaki T, Suzuki SO, Iwaki T, Kobayashi K, Mihara F, Honda H (2008) Perfusion imaging of brain tumors using arterial spin-labeling: correlation with histopathologic vascular density. Am J Neuroradiol 29: 688-693. doi:10.3174/ajnr.A0903

25. Terakawa Y, Tsuyuguchi N, Iwai Y, Yamanaka K, Higashiyama S, Takami T, Ohata K (2008) Diagnostic accuracy of ${ }^{11} \mathrm{C}$ methionine PET for differentiation of recurrent brain tumors from radiation necrosis after radiotherapy. J Nucl Med 49:694-699. doi:10.2967/jnumed.107.048082

26. Popperl G, Gotz C, Rachinger W, Gildehaus FJ, Tonn JC, Tatsch $\mathrm{K}$ (2004) Value of O-(2-[ $\left.{ }^{18} \mathrm{~F}\right]$ fluoroethyl)-L-tyrosine PET for the diagnosis of recurrent glioma. Eur J Nucl Med Mol Imaging 31:1464-1470. doi:10.1007/s00259-004-1590-1

27. Herzog H, Langen KJ, Weirich C, Rota Kops E, Kaffanke J, Tellmann L, Scheins J, Neuner I, Stoffels G, Fischer K, Caldeira L, Coenen HH, Shah NJ (2011) High resolution BrainPET combined with simultaneous MRI. Nuklearmedizin 50:74-82. doi:10.3413/Nukmed-0347-10-09

28. Neuner I, Kaffanke JB, Langen KJ, Kops ER, Tellmann L, Stoffels G, Weirich C, Filss C, Scheins J, Herzog H, Shah NJ (2012) Multimodal imaging utilising integrated MR-PET for human brain tumour assessment. Eur Radiol. doi:10.1007/ s00330-012-2543-x

29. Boss A, Bisdas S, Kolb A, Hofmann M, Ernemann U, Claussen CD, Pfannenberg C, Pichler BJ, Reimold M, Stegger L (2010) Hybrid PET/MRI of intracranial masses: initial experiences and comparison to PET/CT. J Nucl Med 51:1198-1205. doi: 10.2967/jnumed.110.074773

30. Schwenzer NF, Stegger L, Bisdas S, Schraml C, Kolb A, Boss A, Muller M, Reimold M, Ernemann U, Claussen CD, Pfannenberg C, Schmidt H (2012) Simultaneous PET/MR imaging in a human brain PET/MR system in 50 patients-current state of image quality. Eur J Radiol. doi:10.1016/j.ejrad.2011.12.027

31. Thorwarth D, Henke G, Muller AC, Reimold M, Beyer T, Boss A, Kolb A, Pichler B, Pfannenberg C (2011) Simultaneous ${ }^{68}$ GaDOTATOC-PET/MRI for IMRT treatment planning for meningioma: first experience. Int J Radiat Oncol Biol Phys 81:277-283. doi:10.1016/j.ijrobp.2010.10.078

32. Thomson V, Pialat JB, Gay F, Coulon A, Voloch A, Granier A, Guerin JC, Viallon M, Berthezene Y (2008) Whole-body MRI for metastases screening: a preliminary study using 3D VIBE sequences with automatic subtraction between noncontrast and contrast enhanced images. Am J Clin Oncol 31:285-292. doi: 10.1097/COC.0b013e31815e3ff4

33. Schmidt GP, Baur-Melnyk A, Herzog P, Schmid R, Tiling R, Schmidt M, Reiser MF, Schoenberg SO (2005) High-resolution whole-body magnetic resonance image tumor staging with the use of parallel imaging versus dual-modality positron emission tomography-computed tomography: experience on a 32-channel system. Invest Radiol 40:743-753 (pii: 00004424-20051200000001)
34. Drzezga A (2009) Diagnosis of Alzheimer's disease with $\left[{ }^{18} \mathrm{~F}\right] \mathrm{PET}$ in mild and asymptomatic stages. Behav Neurol 21:101115. doi:10.3233/BEN-2009-0228

35. Dukart J, Mueller K, Horstmann A, Barthel H, Moller HE, Villringer A, Sabri O, Schroeter ML (2011) Combined evaluation of FDG-PET and MRI improves detection and differentiation of dementia. PLoS One 6:e18111. doi:10.1371/journal.pone. 0018111

36. Kantarci K, Lowe VJ, Boeve BF, Weigand SD, Senjem ML, Przybelski SA, Dickson DW, Parisi JE, Knopman DS, Smith GE, Ferman TJ, Petersen RC, Jack CR Jr (2011) Multimodality imaging characteristics of dementia with Lewy bodies. Neurobiol Aging 33(9):2091-2105. doi:10.1016/j.neurobiolaging. 2011.09.024

37. Kumar A, Semah F, Chugani HT, Theodore WH (2012) Epilepsy diagnosis: positron emission tomography. Handb Clin Neurol 107:409-424. doi:10.1016/B978-0-444-52898-8.00026-4

38. Salamon N, Kung J, Shaw SJ, Koo J, Koh S, Wu JY, Lerner JT, Sankar R, Shields WD, Engel J Jr, Fried I, Miyata H, Yong WH, Vinters HV, Mathern GW (2008) FDG-PET/MRI coregistration improves detection of cortical dysplasia in patients with epilepsy. Neurology 71:1594-1601. doi:10.1212/01.wnl.0000334752.418 $07.2 \mathrm{f}$

39. Madan N, Grant PE (2009) New directions in clinical imaging of cortical dysplasias. Epilepsia 50(Suppl 9):9-18. doi:10.1111/ j.1528-1167.2009.02292.x

40. Varrone A, Asenbaum S, Vander Borght T, Booij J, Nobili F, Nagren K, Darcourt J, Kapucu OL, Tatsch K, Bartenstein P, Van Laere K (2009) EANM procedure guidelines for PET brain imaging using $\left[{ }^{18} \mathrm{~F}\right] \mathrm{FDG}$, version 2. Eur J Nucl Med Mol Imaging 36:2103-2110. doi:10.1007/s00259-009-1264-0

41. Kleis M, Daldrup-Link H, Matthay K, Goldsby R, Lu Y, Schuster T, Schreck C, Chu PW, Hawkins RA, Franc BL (2009) Diagnostic value of PET/CT for the staging and restaging of pediatric tumors. Eur J Nucl Med Mol Imaging 36:23-36. doi:10.1007/ s00259-008-0911-1

42. Punwani S, Taylor SA, Bainbridge A, Prakash V, Bandula S, De Vita E, Olsen OE, Hain SF, Stevens N, Daw S, Shankar A, Bomanji JB, Humphries PD (2010) Pediatric and adolescent lymphoma: comparison of whole-body STIR half-Fourier RARE MR imaging with an enhanced PET/CT reference for initial staging. Radiology 255:182-190. doi:10.1148/radiol.09091105

43. Zaidi H, Ruest T, Schoenahl F, Montandon ML (2006) Comparative assessment of statistical brain MR image segmentation algorithms and their impact on partial volume correction in PET. Neuroimage 32:1591-1607 (pii: S1053-8119(06)00579-9)

44. Chetelat G, Desgranges B, Landeau B, Mezenge F, Poline JB, de la Sayette V, Viader F, Eustache F, Baron JC (2008) Direct voxelbased comparison between grey matter hypometabolism and atrophy in Alzheimer's disease. Brain 131:60-71 (pii: awm288)

45. Lovblad KO, Haller S, Pereira VM (2012) Stroke: high-field magnetic resonance imaging. Neuroimaging Clin $\mathrm{N}$ Am 22:191-205. doi:10.1016/j.nic.2012.02.002

46. Spaeth N, Wyss MT, Pahnke J, Biollaz G, Lutz A, Goepfert K, Westera G, Treyer V, Weber B, Buck A (2006) Uptake of ${ }^{18} \mathrm{~F}$ fluorocholine, ${ }^{18}$ F-fluoro-ethyl-L: -tyrosine and ${ }^{18}$ F-fluoro-2deoxyglucose in F98 gliomas in the rat. Eur J Nucl Med Mol Imaging 33:673-682. doi:10.1007/s00259-005-0045-7

47. Catana C, Benner T, van der Kouwe A, Byars L, Hamm M, Chonde DB, Michel CJ, El Fakhri G, Schmand M, Sorensen AG (2011) MRI-assisted PET motion correction for neurologic studies in an integrated MR-PET scanner. J Nucl Med 52:154-161. doi:10.2967/jnumed.110.079343

48. Chonde DB, Arabasz G, Catana C (2012) Evaluation of motion correction schemes for simultaneous FDG-PET/MR studies in Alzheimer's disease. J Nucl Med 53:208 
49. Boss A, Kolb A, Hofmann M, Bisdas S, Nagele T, Ernemann U, Stegger L, Rossi C, Schlemmer HP, Pfannenberg C, Reimold M, Claussen CD, Pichler BJ, Klose U (2010) Diffusion tensor imaging in a human PET/MR hybrid system. Invest Radiol 45:270-274. doi:10.1097/RLI.0b013e3181dc3671

50. Kolb A, Wehrl HF, Hofmann M, Judenhofer MS, Eriksson L, Ladebeck R, Lichy MP, Byars L, Michel C, Schlemmer HP, Schmand M, Claussen CD, Sossi V, Pichler BJ (2012) Technical performance evaluation of a human brain PET/MRI system. Eur Radiol 22:1776-1788. doi:10.1007/s00330-012-2415-4

51. Weirich C, Brenner D, Scheins J, Besancon E, Tellmann L, Herzog H, Shah NJ (2012) Analysis and correction of count rate reduction during simultaneous MR-PET measurements with the BrainPET scanner. IEEE Trans Med Imaging 31:1372-1380. doi: 10.1109/TMI.2012.2188903
52. Martinez-Moller A, Nekolla SG (2012) Attenuation correction for PET/MR: problems, novel approaches and practical solutions. Z Med Phys 22(4):299-310. doi:10.1016/j.zemedi.2012.08.003

53. Catana C, van der Kouwe A, Benner T, Michel CJ, Hamm M, Fenchel M, Fischl B, Rosen B, Schmand M, Sorensen AG (2010) Toward implementing an MRI-based PET attenuation-correction method for neurologic studies on the MR-PET brain prototype. J Nucl Med 51:1431-1438. doi:10.2967/jnumed.109.069112

54. Malone IB, Ansorge RE, Williams GB, Nestor PJ, Carpenter TA, Fryer TD (2011) Attenuation correction methods suitable for brain imaging with a PET/MRI scanner: a comparison of tissue atlas and template attenuation map approaches. J Nucl Med 52:1142-1149. doi:10.2967/jnumed.110.085076 